\title{
DIATOMIC MOLECULE AS A DEFORMABLE BODY*
}

\author{
M. MoLSKI \\ Department of Theoretical Chemistry, Faculty of Chemistry, A. Mickiewicz University \\ Grunwaldzka 6, 60-780 Poznan, Poland
}

(Received January 21, 1992; in final form March 30, 1992)

\begin{abstract}
On the basis of the deformable body model and harmonic potential approximation, a nonlinear Hamiltonian describing rovibrational states of diatomic molecules has been derived. The proposed approach is extended to include the Simons-Parr-Finlan potential, and the obtained equations are applied in evaluation of molecular constants and in reproduction of rovibrational spectra of the ${ }^{1} \Sigma$ state of ${ }^{12} \mathrm{C}^{32} \mathrm{~S},{ }^{13} \mathrm{C}^{16} \mathrm{O}$ and rotational spectra of ${ }^{12} \mathrm{C}^{16} \mathrm{O}$ molecules. A comparison with the standard Dunham results is also made.
\end{abstract}

PACS numbers: $33.10 . \mathrm{Cs}, 33.10 . \mathrm{Jz}$

\section{Introduction}

Investigation of systems with rotational and vibrational degrees of freedom (the so-called rovibrational systems) has been performed in the framework of theories which are built up on the basis of rigid, semirigid or nonrigid models of a molecule [1]. Recent research in this field brought forth a few significant results [2-9] providing alternative solutions to the problem of description of rovibrational systems and interpretation of molecular spectra. In particular, a soft body model $[8,9]$ in which the interatomic distances depend on the angular frequency of rotation, has been introduced. As a result, the generalized Darling-Dennison Hamiltonian was obtained, yielding quite satisfactory relationship between theoretical and experimental values of rotational energy of diatomic molecules. Another proposal is the deformable body model [10], describing a molecule whose element positions depend on the momentum and angular momentum as a result of the deformational influence of the Coriolis and centrifugal forces acting in the rovibrational systems. Taking into account the above assumption and the harmonic potential approximation; a nonlinear (in the quantum-mechanical sense) Hamiltonian was derived, which has not as yet been used in the rovibrational spectroscopy.

*This research was supported by KBN grant 2066391.01 . 
In the context of the above consideration, a general aim of this paper is to derive, in the framework of the deformable body model and harmonic potential approximation, a nonlinear quantum-mechanical Hamiltonian describing rovibrational states of diatomic molecules. Next, the proposed approach will be extended to include a more complicated case of the Simons-Parr-Finlan potential, and the obtained equations will be applied in evaluation of the molecular constants and in reproduction of rovibrational spectra of the ${ }^{1} \Sigma$ state of ${ }^{12} \mathrm{C}^{32} \mathrm{~S},{ }^{13} \mathrm{C}^{16} \mathrm{O}$, and rotational spectra of ${ }^{12} \mathrm{C}^{16} \mathrm{O}$ molecules.

\section{Nonlinear Hamiltonian of diatomic molecules in harmonic potentiall approximation}

Let us recall the fundamental assumptions for the deformable model of molecules:

(i) The centrifugal and Coriolis forces operate in the rovibrational systems.

(ii) The displacements observed in the rovibrational systems are of vibrational-deformational type, i.e., vibrations occur in the potential force field modified by the centrifugal and Coriolis forces.

(iii) The magnitude of the deformational displacements of atoms determines the dynamical equilibrium state between the deforming and restoring potential forces.

The application of the deformable body model in description of rovibrational systems leads to the nonlinear [10] (in the quantum-mechanical sense) Hamiltonian

$$
\begin{aligned}
& \hat{H}=\frac{1}{2} \hat{\boldsymbol{P}}^{T} \boldsymbol{K}_{0}^{-1} \hat{\boldsymbol{P}}-\frac{1}{8} \hbar^{2} \operatorname{Tr} \mu_{0}+\frac{1}{2} \eta^{T}(\hat{\boldsymbol{B}}+\boldsymbol{\Lambda}) \boldsymbol{\eta}-\frac{1}{8} \hat{\boldsymbol{A}}^{T}(\hat{\boldsymbol{B}}+\boldsymbol{\Lambda})^{-1} \hat{\boldsymbol{A}}, \\
& \hat{\boldsymbol{A}}=\left\{\hat{\boldsymbol{P}}^{T} \boldsymbol{K}_{k}^{-1} \hat{\boldsymbol{P}}-\frac{1}{8} \hbar^{2} \operatorname{Tr} \mu_{k}\right\} \\
& \hat{\boldsymbol{B}}=\left\{\hat{\boldsymbol{P}}^{T} \boldsymbol{K}_{k s^{-1}} \hat{\boldsymbol{P}}-\frac{1}{8} \hbar^{2} \operatorname{Tr} \mu_{k s}\right\}, \\
& \operatorname{Tr} \boldsymbol{\mu}=\sum_{\alpha} \mu_{\alpha \alpha}, \quad \alpha=x, y, z,
\end{aligned}
$$

where $\hat{\boldsymbol{P}}=\{\hat{\boldsymbol{J}}, \hat{\boldsymbol{p}}\}$ and $\hat{\boldsymbol{J}}, \hat{\boldsymbol{p}}$ are the operators of the angular and vibrational momenta, $\boldsymbol{\Lambda}$ is the matrix of force constants, and

$$
\boldsymbol{\eta}=\boldsymbol{q}-\hat{\boldsymbol{q}}^{\mathrm{D}}, \quad \hat{\boldsymbol{q}}^{\mathrm{D}}=-\frac{1}{2} \hat{\boldsymbol{A}}(\hat{\boldsymbol{B}}+\boldsymbol{\Lambda})^{-1},
$$

are the matrices of effective normal coordinates and deformational displacements [10], respectively. The remaining matrices occurring in Eqs. (1), (2) and (3) are defined in [10].

Now, let us consider a two-atom system whose components are endowed with masses $m_{1}$ and $m_{2}$. The reduced mass of the system is $m=m_{1} m_{2} /\left(m_{1}+m_{2}\right)$, and the vibrational-deformational displacements are described by the coordinate $q$ canonically coupled with the momentum $p$. When the Taylor expansion of the reciprocal moment of inertia in the equilibrium configuration $q_{0}$ is employed

$$
\left[m\left(q_{0}+q\right)^{2}\right]^{-1}=\left(m q_{0}^{2}\right)^{-1}-2\left(m q_{0}^{3}\right)^{-1} q+3\left(m q_{0}^{4}\right)^{-1} q^{2}-\ldots,
$$


Hamiltonian (1) for a two-atom system reduces to the simple form

$$
\begin{aligned}
& \hat{H}=\frac{1}{2 m} \hat{\boldsymbol{p}}^{2}+\frac{1}{2}(\hat{\boldsymbol{B}}+\lambda) \eta^{2}+\frac{1}{2} m q_{0}^{2-1} \hat{\boldsymbol{J}}^{2}-\frac{1}{8} \frac{\hat{\boldsymbol{A}}^{2}}{(\hat{\boldsymbol{B}}+\lambda)}, \\
& \hat{\boldsymbol{A}}=-2\left(m q_{0}^{3}\right)^{-1} \hat{\boldsymbol{J}}^{2}, \quad \hat{\boldsymbol{B}}=3\left(m q_{0}^{4}\right)^{-1} \hat{\boldsymbol{J}}^{2},
\end{aligned}
$$

and the substitution of the explicit form of operators $\hat{\boldsymbol{p}}=-\mathrm{i} \hbar \partial / \partial \eta[10], \hat{\boldsymbol{A}}$ and $\hat{\boldsymbol{B}}$ yields

$$
\begin{aligned}
\hat{H}= & -\frac{\hbar^{2}}{2 m} \frac{\partial^{2}}{\partial \eta^{2}}+\frac{1}{2}\left[3\left(m q_{0}^{4}\right)^{-1} \hat{\boldsymbol{J}}^{2}+\lambda\right] \eta^{2}+\frac{1}{2}\left(m q_{0}^{2}\right)^{-1} \hat{\boldsymbol{J}}^{2} \\
& -\frac{1}{2}\left(m q_{0}^{3}\right)^{-2} \hat{\boldsymbol{J}}^{4}\left[3\left(m q_{0}^{4}\right)^{-1} \hat{\boldsymbol{J}}^{2}+\lambda\right]^{-1} .
\end{aligned}
$$

In order to solve the Schrödinger equation with the nonlinear operator (9) we propose the procedure which involves the following steps:

(i) Calculation of the "matrix" element $\langle v, J|\hat{\boldsymbol{B}}+\lambda| v, J\rangle$ on the base of harmonic oscillator and rigid rotor wave functions of the two-atom system.

(ii) Replacement of the nonlinear term $(\hat{\boldsymbol{B}}+\lambda)^{-1}$ in the Hamiltonian by the "matrix" element calculated in (i).

(iii) Averaging of the linear Hamiltonian over rotational states, on the base of rotational wave functions of diatomic molecules.

(iv) Solution of the vibrational Schrödinger equation by using the standard methods of quantum mechanics.

Realization of the above operations yields the Schrödinger equation for harmonic oscillator, which can be strictly solved

$$
\begin{aligned}
& \left\{-\frac{\hbar^{2}}{2 m} \frac{\partial^{2}}{\partial \eta^{2}}+\frac{1}{2} \lambda[1+3 C J(J+1)] \eta^{2}\right. \\
& \left.+B J(J+1)\left[1-\frac{C J(J+1)}{1+3 C J(J+1)}\right]-E_{v J}\right\} \psi_{v J}(\eta)=0, \\
& E_{v J}=\omega_{J}(v+1 / 2)+B J(J+1)\left[\frac{1-C J(J+1)}{1+3 C J(J+1)}\right] \\
& \omega_{J}=\omega[1+3 C J(J+1)]^{1 / 2}, \quad \omega=\hbar\left(\lambda m^{-1}\right)^{1 / 2}, \\
& B=\hbar^{2}\left(2 m q_{0}^{2}\right)^{-1}, \quad C=\hbar^{2}\left(m \lambda q_{0}^{4}\right)^{-1}, \\
& \psi_{v J}=N_{v J} \exp \left(-1 / 2 \gamma_{J} \eta^{2}\right) H_{v J}\left(\gamma_{J}^{1 / 2} \eta\right), \quad \gamma_{J}=m \hbar^{-1} \omega_{J},
\end{aligned}
$$

where $H_{v J}\left(\gamma_{J}^{1 / 2} \eta\right)$ is Hermite polynomial in $\gamma_{J}^{1 / 2} \eta$. 
The rovibrational transitions $v \rightarrow v+1, J \rightarrow J+1$, new equilibrium distance $q_{J}=q_{0}+\dot{q}^{\mathbf{D}}$ and modified force constant $\lambda_{J}$ can be calculated from the following formulae:

$$
\begin{aligned}
& \Delta E_{v J}=\omega[1+3 C(J+1)(J+2)]^{1 / 2}(v+3 / 2)-\omega[1+3 C J(J+1)]^{1 / 2} \\
& \times(v+1 / 2) Q+2 B(J+1)-\frac{B C(J+1)^{2}(J+2)^{2}}{1+3 C(J+1)(J+2)}+\frac{B C J^{2}(J+1)^{2}}{1+3 C J(J+1)} \\
& q_{J}=q_{0}+2(B C / \lambda)^{1 / 2}[1+3 C J(J+1)]^{-1} \\
& \lambda_{J}=\lambda[1+3 C J(J+1)]
\end{aligned}
$$

whereas the rotational energy in the vibrational state $v$ and the energy of rotational transition are described by the equations

$$
\begin{aligned}
& E_{v J}=\omega[1+3 C J(J+1)]^{1 / 2}(v+1 / 2)+B J(J+1) \\
& \quad-\frac{B C J^{2}(J+1)^{2}}{1+3 C J(J+1)}-E_{v 0}, \\
& E_{v 0}=\omega(v+1 / 2), \\
& \Delta E_{v J}=\omega[1+3 C(J+1)(J+2)]^{1 / 2}(v+1 / 2)-\omega[1+3 C J(J+1)]^{1 / 2} \\
& \times(v+1 / 2)+2 B(J+1)-\frac{B C(J+1)^{2}(J+2)^{2}}{1+3 C(J+1)(J+2)}+\frac{B C J^{2}(J+1)^{2}}{1+3 C J(J+1)},
\end{aligned}
$$

where $E_{v 0}$ is the vibrational energy in the rotational state $J=0$. Taking into account the general definition (5), it is apparent that $\eta=q-2(B C / \lambda)^{1 / 2}[1+$ $3 C J(J+1)]^{-1}$. It is interesting to note that identical formulas for effective vibrational coordinates and rovibrational energy (11) were obtained by Schrödinger [11], who, however, has not given any physical interpretation of the transition to new variable $q \rightarrow \eta$.

\section{Nonlinear Hamiltonian of diatomic molecules in Simons-Parr-Finlan potential approximation}

The results obtained in the previous section indicate that in rovibrational two-atom systems the vibrations occur in the harmonic force field with the modified harmonic constant and the changed equilibrium configuration $q_{J}$. It is a simple consequence of the deformational action of the centrifugal forces which operate in all systems with a rotational degree of freedom [10]. In view of the above, $q^{D}$ may be interpreted as a deformational displacement resulting from the centrifugal distortion, and $\eta=q-q^{\mathbf{D}}$ is an effective coordinate describing vibrations around the new equilibrium configuration $q_{J}$. The above interpretation permits a modification of the presented approach to two-atom systems by using the expansion of the potential and rotational term occurring in the Schrödinger equation

$$
\left(-\frac{\hbar^{2}}{2 m} \frac{\partial^{2}}{\partial q^{2}}+\frac{\hbar^{2} J(J+1)}{2 m\left(q_{0}+q\right)^{2}}+U(q)-E_{v J}\right) \psi_{v J}=0
$$


in the changed equilibrium point $q_{J}$ instead of $q_{0}$

$$
\begin{aligned}
& U_{\mathrm{ef}}(q)=\frac{\hbar^{2} J(J+1)}{2 m\left(q_{0}+q\right)^{2}}+U(q)=U\left(q^{\mathrm{D}}\right)+\frac{\hbar^{2} J(J+1)}{2 m\left(q_{0}+q^{\mathrm{D}}\right)^{2}} \\
& +\left[\frac{\partial U(q)}{\partial q}-\frac{\hbar^{2} J(J+1)}{m\left(q_{0}+q\right)^{3}}\right]_{q^{\mathrm{D}}}\left(q-q^{\mathrm{D}}\right) \\
& +\frac{1}{2}\left[\frac{\partial^{2} U(q)}{\partial q^{2}}+\frac{3 \hbar^{2} J(J+1)}{m\left(q_{0}+q\right)^{4}}\right]_{q^{\mathrm{D}}}\left(q-q^{\mathrm{D}}\right)^{2}+\ldots
\end{aligned}
$$

The linear effective force constant $f_{J}=\left(\partial U_{\mathrm{ef}}(q) / \partial q\right)_{q} \mathrm{D}$ is identically equal to zero because the potential function $U_{\text {ef }}(q)$ has a minimum in the changed equilibrium configuration. In view of the above, the wave equation (20) for a parabolic expansion may be replaced by the set of equations

$$
\begin{aligned}
& {\left[-\frac{\hbar^{2}}{2 m} \frac{\partial^{2}}{\partial q^{2}}+U_{\mathrm{ef}}\left(q^{\mathrm{D}}\right)+\frac{1}{2} \lambda_{J}\left(q-q^{\mathrm{D}}\right)^{2}-E_{v J}\right] \psi_{v J}=0,} \\
& {\left[\frac{\partial U_{\mathrm{ef}}(q)}{\partial q}\right]_{q^{\mathrm{D}}}=0,} \\
& U_{\mathrm{ef}}\left(q^{\mathrm{D}}\right)=U\left(q^{\mathrm{D}}\right)+\frac{\hbar^{2} J(J+1)}{2 m\left(q_{0}+q^{D}\right)^{2}}, \\
& \lambda_{J}=\left[\frac{\partial^{2} U(q)}{\partial q^{2}}+\frac{3 \hbar^{2} J(J+1)}{m\left(q_{0}+q\right)^{4}}\right]_{q^{\mathrm{D}}},
\end{aligned}
$$

or taking advantage of a new effective vibrational variable $\eta=q-q^{\mathrm{D}}$ in the equivalent form

$$
\left[-\frac{\hbar^{2}}{2 m} \frac{\partial^{2}}{\partial \eta^{2}}+U_{\mathrm{ef}}\left(q^{\mathrm{D}}\right)+\frac{1}{2} \lambda_{J} \eta^{2}-E_{v J}\right]=0 .
$$

All the terms in (22) have clear physical interpretation, namely, $U_{\mathrm{ef}}\left(q^{\mathrm{D}}\right)$ represents an effective rotational energy including a change of the molecular moment of inertia and of the potential energy due to the deformational action of the centrifugal force, whereas the remaining terms describe the energy of a harmonic osciliator with the changed force constant. The auxiliary equation (23) determines the dynamical equilibrium state of the deformational and restoring potential forces, and can be used to calculate the quantity $q^{\mathrm{D}}$. Needles to mention that the wave equation (22) can be analytically solved for any potential $U(q)$, not only for one of harmonic-type.

As an example, let us consider a potential in the simple Simons-Parr-Finlan form [12], i.e.,

$$
U(q)=D_{0}\left(1-\frac{Q_{0}}{Q}\right)^{2}, \quad Q=q_{0}+q, \quad Q_{0}=q_{0},
$$

where $D_{0}$ is a constant related to the dissociation energy of a molecule. Introducing (26a) into (23) and $(24 a, b)$ one gets

$$
\begin{aligned}
& 2 D_{0} Q_{0}\left\{\frac{Q_{J}-Q_{0}\left[1+\hbar^{2} J(J+1)\left(2 m D_{0} Q_{0}^{2}\right)\right]^{-1}}{\left(Q_{J}\right)^{3}}\right\}=0, \\
& U_{\mathrm{ef}}\left(Q_{J}\right)=D_{0}\left(1-\frac{Q_{0}}{Q_{J}}\right)^{2}+\frac{\hbar^{2} J(J+1)}{2 m\left(Q_{J}\right)^{2}}
\end{aligned}
$$




$$
\lambda_{J}=2 D_{0} Q_{0}\left[\frac{3 Q_{0}-2 Q_{J}+3 \hbar^{2} J(J+1)\left(2 D_{0} Q_{0}\right)^{-1}}{\left(Q_{J}\right)^{4}}\right] .
$$

A look into (27) reveals that

$$
Q_{J}=Q_{0}\left[1+\frac{\hbar^{2} J(J+1)}{2 m D_{0} Q_{0}^{2}}\right]
$$

and as a consequence we obtain

$$
\begin{aligned}
& U_{\mathrm{ef}}\left(Q_{J}\right)=\frac{\hbar^{2} J(J+1)}{2 m Q_{0}^{2}\left[1+\hbar^{2} J(J+1)\left(2 m D_{0} Q_{0}^{2}\right)^{-1}\right]}, \\
& \lambda_{J}=\frac{2 D_{0} Q_{0}^{-2}}{\left[1+\hbar^{2} J(J+1)\left(2 m D_{0} Q_{0}^{2}\right)^{-1}\right]^{3}} .
\end{aligned}
$$

Expanding the potential function (26a) in a power series of $q$ for $q / q_{0} \ll 1$

$$
D_{0}\left(\frac{q}{q+q_{0}}\right)^{2}=\left(D_{0} / q_{0}^{2}\right) q^{2}-2\left(D_{0} / q_{0}^{3}\right) q^{3}+\ldots
$$

the quantity $2 D_{0} Q_{0}^{-2}$ which appears in (32) may be interpreted as a force constant $\lambda$. In view of the above, the final form of the wave equation in the parabolic approximation, for a two-atom system described by the Simons-Parr-Finlan potential, may be written as follows:

$$
\left\{-\frac{\hbar^{2}}{2 m} \frac{\partial^{2}}{\partial \eta^{2}}+\frac{B J(J+1)}{1+C J(J+1)}+\frac{1}{2} \lambda[1+C J(J+1)]^{-3} \eta^{2}-E_{v J}\right\}=0
$$

where the eigenvalues and corresponding wave functions are

$$
\begin{aligned}
& E_{v J}=\hbar \omega_{J}(v+1 / 2)+\frac{B J(J+1)}{1+C J(J+1)}, v=0,1,2 \ldots J=0,1,2 \ldots, \\
& \omega_{J}=\left(\lambda m^{-1}\right)^{1 / 2}[1+C J(J+1)]^{3 / 2} \\
& \psi_{v J}=N_{v J} \exp \left(-1 / 2 \gamma_{J} \eta^{2}\right) H_{v J}\left(\gamma_{J}^{1 / 2} \eta\right), \quad \gamma_{J}=m \hbar^{-1} \omega_{J} .
\end{aligned}
$$

\section{Applications}

In order to compare formulae (11) and (35) with the result of the standard Dunham approach [13], let us consider a polynomial expansion of the rovibrational energy in series of vibrational and rotational quantum numbers $v$ and $J$, respectively

$$
\begin{aligned}
E_{v J} & =\sum_{k, l} Y_{k l}(v+1 / 2)^{k} J^{l}(J+1)^{l}, \\
E_{v J}^{\mathrm{H}} & =\hbar \omega(v+1 / 2)\left[1+3 / 2 C J(J+1)-9 / 8 C^{2} J^{2}(J+1)^{2}+\ldots\right] \\
& +B J(J+1)\left[1-C J(J+1)+3 C^{2} J^{2}(J+1)^{2}-\ldots\right],
\end{aligned}
$$




$$
\begin{gathered}
E_{v J}^{\mathrm{SPF}}=\hbar \omega(v+1 / 2)\left[1+3 / 2 C J(J+1)+3 / 8 C^{2} J^{2}(J+1)^{2}-\ldots\right] \\
+B J(J+1)\left[1-C J(J+1)+1 / 2 C^{2} J^{2}(J+1)^{2}-\ldots\right],
\end{gathered}
$$

where $Y_{k l}$ are Dunham parameters whose values are obtained by fitting the experimental data. A comparison of suitable quantities leads to the set of correspondences given for $J=0,1,2$

$$
\begin{aligned}
& Y_{10}=\hbar \omega, \quad Y_{11}=3 / 2 C \hbar \omega, \quad Y_{12}=-9 / 8 C^{2} \hbar \omega, \\
& (41 a, b, c) \\
& Y_{01}=B, \quad Y_{02}=-C B, \quad Y_{03}=3 C^{2} B,
\end{aligned}
$$

for harmonic approximation, and

$$
\begin{array}{lll}
Y_{10}=\hbar \omega, & Y_{11}=3 / 2 C \hbar \omega, & Y_{12}=3 / 8 C^{2} \hbar \omega, \\
Y_{01}=B, & Y_{02}=-C B, & Y_{03}=1 / 2 C^{2} B,
\end{array}
$$

for the model based on the Simons-Parr-Finlan potential. The apparent absence of higher order vibrational terms, is a result of parabolic approximation which eliminates all the terms for $k>1$. On the other hand, there is no limitation on the rotational series, which is fully reproduced. In view of the above, formulae (11) and (35) can be applied to evaluate molecular parameters $\omega, B, C$ and reproduce the energy of rovibrational transitions in a wide range of rotational states of low vibrationally excitied $(v=0,1)$ molecules. As an example molecules ${ }^{12} \mathrm{C}^{32} \mathrm{~S}$ and ${ }^{13} \mathrm{C}^{16} \mathrm{O}$ in the ${ }^{1} \Sigma$ electronic state will be taken into consideration.

In order to compare rovibrational formulae (11) and (35) we will also calculate molecular parameters $q_{0}, \lambda$, and the energy of rotational transitions in the ${ }^{1} \Sigma$ state of ${ }^{12} \mathrm{C}^{16} \mathrm{O}$ molecule. The obtained results and their comparison with the 2and 3-parametric Dunham fit are presented in Table I.

TABLE I

Ground state molecular parameters for ${ }^{12} \mathrm{C}^{16} \mathrm{O}$ fitted to 8 experimental frequencies.

\begin{tabular}{c|c|l|l|c|c|c}
\hline \hline & $\sigma^{a}(\mathrm{MHz})$ & $\lambda\left(\mathrm{Nm}^{-1}\right)$ & $q_{0}(\AA)$ & $\omega\left(\mathrm{cm}^{-1}\right)$ & $B\left(\mathrm{~cm}^{-1}\right)$ & $D\left(\mathrm{~cm}^{-1}\right)$ \\
\hline$I^{b}$ & 0.121 & $1858.43(17)$ & $1.13240187(4)$ & 2144.87195 & 1.917387 & $6.1289 \times 10^{-6}$ \\
$I I^{c}$ & 0.042 & $1883.063(41)$ & $1.12937113(4)$ & 2159.03925 & 1.927692 & $6.1468 \times 10^{-6}$ \\
$I I I^{d}$ & 0.047 & 1876.540 & 1.1308868 & 2155.26610 & 1.922528 & $6.1187 \times 10^{-6}$ \\
$I V^{e}$ & 0.050 & & 1.128323 & 2169.81358 & 1.93128087 & $6.1214 \times 10^{-6}$ \\
\hline
\end{tabular}

a Standard deviation of the fit.

${ }^{b} \lambda$ and $q_{0}$ are calculated from (11), whereas remaining parameters from (12b), (13a) and relation $D=4 B^{3} \omega^{-2}$.

c $\lambda$ and $q_{0}$ are calculated from (35), whereas remaining parameters from (12b), (13a) and relation $D=4 B^{3} \omega^{-2}$.

${ }^{d}$ Parameters calculated from Dunham formula $E_{v J}=J(J+1)[B-D J(J+1)]$.

experimental data from [15]. 
TABLE II

Ground state molecular constants $\left(\mathrm{cm}^{-1}\right)$ for ${ }^{12} \mathrm{C}^{32} \mathrm{~S}$ fitted to 13 experimental frequencies.

\begin{tabular}{c|c|l|l|c|c|c}
\hline \hline & \multicolumn{1}{|c|}{$\sigma^{a}$} & \multicolumn{1}{c|}{$\omega$} & \multicolumn{1}{c|}{$B$} & $C^{b} \times 10^{-6}$ & $D \times 10^{-5}$ & $\alpha \times 10^{-3}$ \\
\hline$I^{c}$ & 0.0020 & $1272.1631(16)$ & $0.82152(21)$ & $-3.2653(95)$ & & \\
$I I^{d}$ & 0.0014 & $1272.1695(15)$ & $0.819194(70)$ & $3.0290(70)$ & & \\
$I I I^{e}$ & 0.0015 & $1272.1653(12)$ & $0.82071(15)$ & & & $6.074(13)$ \\
$I V^{f}$ & 0.0329 & $1272.261(23)$ & $0.7880(18)$ & & $-4.69(23)$ & \\
$V$ & & $1272.1663(15)^{g}$ & $0.8200462^{h}$ & & $14.3^{h}$ & 5.9224 \\
\hline
\end{tabular}

a Standard deviation of the fit.

${ }^{b}$ Parameter $C$ is dimensionless.

${ }^{c}$ Parameters calculated from (11).

${ }^{d}$ Parameters calculated from (35).

e Energy calculated from Dunham formula $E_{v J}=\omega(v+1 / 2)+J(J+1)[B-\alpha(v+1 / 2)]$.

${ }^{f}$ Energy calculated from Dunham formula $E_{v} \jmath=\omega(v+1 / 2)+J(J+1)[B-D J(J+1)]$.

${ }^{g}$ Experimental data from [14].

${ }^{h}$ Experimental data from [15].

TABLE III Ground state molecular constants $\left(\mathrm{cm}^{-1}\right)$ for ${ }^{12} \mathrm{C}^{16} \mathrm{O}$ fitted to 35 experimental frequencies.

\begin{tabular}{|c|c|c|c|c|c|}
\hline & $\sigma^{a}$ & $\omega$ & $B$ & $C^{b} \times 10^{-6} D \times 10^{-6}$ & $\alpha \times 10^{-2}$ \\
\hline$I^{c}$ & 0.0438 & $2095.945(23)$ & $1.8672(15)$ & $-5.974(23)$ & \\
\hline$I I^{d}$ & 0.0018 & $2096.0898(31)$ & $1.84206(19)$ & $5.0279(26)$ & \\
\hline$I I I^{e}$ & 0.0244 & $2095.999(13)$ & $1.85766(79)$ & & $1.766155(38)$ \\
\hline$I V^{f}$ & 0.3011 & $2097.01(14)$ & $1.6970(58)$ & $74.0(20)$ & \\
\hline$V^{g}$ & & $2121.43956(27)$ & $1.84615215(39)$ & $5.59504(177)$ & $1.6360222(663)$ \\
\hline
\end{tabular}

a Standard deviation of the fit.

${ }^{b}$ Parameter $C$ is dimensionless.

c Parameters calculated from (11).

${ }^{d}$ Parameters calculated from (35).

e Parameters calculated from Dunham formula $E_{v J}=\omega(v+1 / 2)+J(J+1)[B-\alpha(v+1 / 2)]$.

$f$ Parameters calculated from Dunham formula $E_{v J}=\omega(v+1 / 2)+J(J+1)[B-D J(J+1)]$.

$g$ Experimental data from [16].

The best values of the molecular parameters will be determined by the linear least-square routine in which the statistical weights, proportional to the inverse of experimental uncertainties, are taken as being equal to one. Tables II and III present the parameters with their sigma standard errors obtained by the fitting procedure and used for the calculation of the rovibrational transitions. Moreover, the calculated frequencies are compared with these obtained applying the 3-parametric Dunham formulae, including two different sets of parameters $\{\omega, \alpha, B\}$ and $\{\omega, B, D\}$. 


\section{Discussion}

An application of the deformable body model has lead to a new expansion of rovibrational energy of diatomic molecules which provides quite satisfactory relationship between theoretical and experimental data in a wide range of rotational states. However, the parabolic approximation limits the application of the derived equations to the low vibrational states $(v=0,1)$ of molecules.

The results obtained indicate that formula (35) reproduces the energy of rovibrational transitions and molecular parameters for all the molecules considered more precisely, than Eq. (11) and 3-parametric Dunham expansions. Because parabolic approximation for vibrationally low excited molecules seems to be relevant for harmonic as well as Simons-Parr-Finlan potential, we arrive at the conclusion that the model assuming vibrational displacements about the modified equilibrium configuration leads to a better physically supported equation than the one considering kinematics in the vicinity of the original configuration. The difference in accuracy of calculations performed by using both formulae is especially apparent for ${ }^{13} \mathrm{C}^{16} \mathrm{O}$, when $\sigma$ changes as much as two orders of magnitude relative to the Dunham result. However, it must be emphasized here that both formulae give unsatisfactory reproduction of the equilibrium distance and force constant for ${ }^{12} \mathrm{C}^{16} \mathrm{O}$, relative to the standard methods based on the polyparametric expansions. It is a consequence of application of very simple model potentials which do not reproduce satisfactorily actual internal motion potential of diatomic systems and lead to the pure parametric equations.

The deformable body model introduces important relations between the standard constants and the new molecular constants connected with the equilibrium distance and potential parameters, thus making it possible to calculate values of these parameters by using the rovibrational spectra of diatomic molecules. Moreover, the derived formulae permit to obtain the effective force constant and changed frequencies of normal vibrations, as well as new equilibrium distances in each of the rotational state. As follows from relations $(41 b, c)$ and $(43 b, c)$, the parameters $Y_{1 J}$ responsible for rovibrational couplings have been taken into account in the derived formulae in a natural way, and the results obtained for ${ }^{12} \mathrm{C}^{32} \mathrm{~S}$ indicate that the constant $Y_{11}$ should be regarded in the calculations, although, it has been disregarded in Ref. [14].

It is well-known that the Dunham expansion of the rovibrational energy of diatomic molecules does not provide any information about the wave functions of individual states of a molecule. In consequence, matrix elements of operators, Franck-Condon factors and intensities of rovibrational transitions, cannot be directly calculated. The method presented here provides the possibility to obtain the energy levels as well as the wave functions in the analytical form, which is not possible within the standard Dunham approach.

The proposed method based on the deformable body model and the parabolic approximation may be developed in the two directions:

(i) Taking into account higher-order terms in the expansion of $U_{\text {ef }}(q)$, and employing the perturbation method for calculation of the rovibrational energy including anharmonic terms. 
(ii) Considering other potential functions, more accurately approximating the actual potential of the system under study.

The results of investigation of the aforementioned problems will be presented in separate papers, however, the results obtained in this work provide the introductory information on the accuracy of calculations and the possible limitations relevant to application of the nonlinear rovibrational Hamiltonian derived on the basis of the deformable body model.

\section{Acknowledgment}

The author is indebted to Professor J. Konarski for stimulating discussions.

\section{References}

[1] D. Papoušek, M.R. Aliev, Molecular Vibrational-Rotational Spectra, Academia, Praga 1982.

[2] A.J. Thakar, J. Chem. Phys. 62, 1693 (1975).

[3] N.S. Mohammad, Int. J. Quantum Chem. 17, 943 (1980).

[4] J.N. Huffaker, J. Chem. Phys. 64, 3175 (1976); 64, 4565 (1976).

[5] Y. Makushin, O.V. Naumienko, O.N. Ulenikow, J. Mol. Spetrosc. 103, 221 (1984).

[6] V.I. Pierevalov, V.G. Tyuterev, B.I. Zhilinski, J. Mol. Spetrosc. 103, 147 (1984).

[7] K.S. Jordan, J.L. Kinsey, R. Silbey, J. Chem. Phys. 61, 911 (1974).

[8] J. Konarski, J. Mol. Spetrosc. 124, 218 (1987).

[9] J. Konarski, Acta Phys. Pol. A74, 235 (1988).

[10] M. Molski, Acta Phys. Pol. A76, 877 (1989).

[11] E. Schrödinger, Ann. Phys. 79, 489 (1926).

[12] G. Simons, R.G. Parr, J.M. Finlan, J. Chem. Phys. 59, 3229 (1973).

[13] J.L. Dunham, Phys. Rev. 41, 713 (1932).

[14] C. Yamada, E. Hirota, J. Mol. Spectrosc. 74, 203 (1979).

[15] K.P. Huber, G. Herzberg, Constants of Diatomic Molecules, Van Nostrad, New York 1979.

[16] D. Chen, K. Narahari Rao, J. Mol. Spetrosc. 61, 71 (1976).

[17] I.G. Nolt, J.V. Radostitz, G. DiLonardo, K.M. Evenson, D.A. Jennings, K.R. Leopold, M.D. Vanek, L.R. Zink, A. Hinz, K.V. Chance, J. Mol. Spetrosc. 125, 274 (1987). 